\title{
WWW'18 Workshop on Exploitation of Social Media for Emergency Relief and Preparedness: Chairs' Welcome
}

It is our great pleasure to welcome you to the WWW 2018 Workshop on Exploitation of Social Media for Emergency Relief and Preparedness (SMERP).

User-generated content on online social media (OSM) platforms has become an important source of real-time information during emergency events. The SMERP workshop series aims to provide a forum for researchers working on utilizing OSM for emergency preparedness and aiding postemergency relief operations. The workshop aims to bring together researchers from diverse fields Information Retrieval, Data Mining and Machine Learning, Natural Language Processing, Social Network Analysis, Computational Social Science, Human Computer Interaction - who can potentially contribute to utilizing social media for emergency relief and preparedness. The first SMERP workshop was held in April 2017 in conjunction with the ECIR 2017 conference. This $2^{\text {nd }}$ SMERP Workshop with The Web Conference 2018 includes two keynote talks, a peer-reviewed research paper track, and a panel discussion. The workshop site is at: https://www.cse.iitk.ac.in/users/kripa/smerp2018/.

The 2018 workshop includes keynote talks by two eminent researchers:

- Utility of Social Media Information in Response to Natural Disasters, by Prasenjit Mitra, Professor of Information Sciences and Technology and Associate Dean for Research, The Pennsylvania State University, USA

- Transforming Social Big Data into Timely Decisions and Actions for Crisis Mitigation and Coordination, by Amit Seth, Professor of Computer Science and Executive Director of Kno.e.sis Centre, Wright State University, USA

The research paper track attracted submissions from the USA, Europe, and Asia. Nine (09) papers were submitted, out of which the following five (05) papers were accepted through a peer-review process, for presentation at the workshop:

- Modeling evacuation behavior of NYC Twitter users during Hurricane Sandy. Dheeraj Kumar and Satish Ukkusuri.

- Flood relevance estimation from visual and textual content in social media streams. Anastasia Moumtzidou, Stelios Andreadis, Ilias Gialampoukidis, Anastasios Karakostas, Stefanos Vrochidis and Yiannis Kompatsiaris.

- Class Specific TF-IDF Boosting for Short Text Classification. Samujjwal Ghosh and Maunendra Sankar Desarkar.

- Gold Standard Creation for Microblog Retrieval: Challenges of Completeness in IRMiDis 2017. Ribhav Soni and Sukomal Pal.

- SAVITR: A System for Real-time Location Extraction from Microblogs during Emergencies. Ritam Dutt, Kaustubh Hiware, Avijit Ghosh and Rameshwar Bhaskaran.

It can be seen that the accepted papers include a good mixture of research that develop algorithms, research that model human behavior during emergencies, and researchon development and deployment of usable systems.

The final session of the workshop includes a panel discussion on the upcoming practical challenges related to use of OSM during emergencies. Some of the discussion points are the applicability of 
models pre-trained over past events during a future event, exploring the combination of OSM with other sources of information for more effective extraction of critical information, utility of geographical knowledge bases, and so on.

As part of the workshop, we are also releasing a dataset containing tweets, news articles, and images related to a particular emergency event (the Nepal earthquake in April 2015). We invite the research community to use the dataset for developing improved algorithms for multi-modal information extraction at the times of emergency.

We hope that you will find the workshop program interesting. Further details about the workshop, as well as the dataset mentioned above, can be found at: https://www.cse.iitk.ac.in/users/kripa/smerp2018/.

$\begin{array}{ll}\begin{array}{l}\text { Debasis Gangully } \\ \text { IBM Research, Ireland }\end{array} & \begin{array}{l}\text { Gareth J. F. Jones } \\ \text { DCU, Ireland }\end{array} \\ \begin{array}{l}\text { Kripabandhu Ghosh } \\ \text { IIT Kanpur, India }\end{array} & \begin{array}{l}\text { Marie-Francine Moens } \\ \text { KU Leuven, Belgium }\end{array} \\ \begin{array}{l}\text { Saptarshi Ghosh } \\ \text { IIT Kharagpur, India }\end{array} & \begin{array}{l}\text { Tanmoy Chalkraborty } \\ \text { IIIT Delhi, India }\end{array}\end{array}$

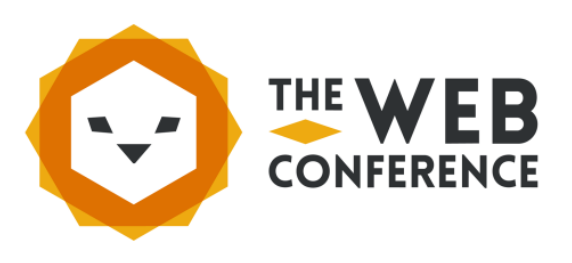

IIT Kharagpur, India

IIIT Delhi, India 


\title{
WWW'18 Workshop on Exploitation of Sociall Media for Emergency Relief and Preparedness: Organization
}

\author{
Organization Committee: Debasis Ganguly (IBM Research Labs, Dublin, Ireland) \\ Gareth J. F. Jones (Dublin City University, Ireland) \\ Kripabandhu Ghosh (IIT Kanpur, India) \\ Marie-Francine Moens (KU Leuven, Belgium) \\ Saptarshi Ghosh (IIT Kharagpur, India) \\ Tanmoy Chakraborty (IIIT Delhi, India)
}

Program Committee: Arnab Bhattacharya (IIT Kanpur, India)

Charles Jochim (IBM Research, Dublin, Ireland)

Dongxiao He (Tianjin University, China)

Douglas W. Oard (University of Maryland, College Park, USA)

Girish Keshav Palshikar (Tata Consultancy Services, India)

Jaegul Chao (Korea University, Korea)

Manish Gupta (Microsoft Research, India)

Monojit Choudhury (Microsoft Research, India)

Muhammad Imran (Qatar Computing Research Institute, Qatar)

Noseong Park (University of North Carolina, Charlotte, USA)

Parantapa Bhattacharya (Virginia Tech, USA)

Parantapa Goswami (Viseo Technologies, Grenoble, France)

Parth Gupta (Amazon, India)

Richard McCreadie (University of Glasgow, UK)

Rishiraj Saharoy (Max Planck Institute for Informatics, Germany)

Srijan Kumar (Stanford University, USA)

Tinne Tuytelaars (KU Leuven, Belgium)

Yufang Hou (IBM Research, Dublin, Ireland) 